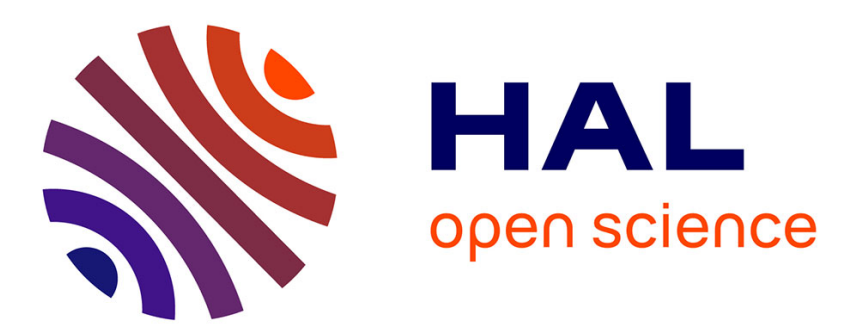

\title{
Alpha decay potential barriers and half-lives and analytical formula predictions for superheavy nuclei \\ Guy Royer, H.F. Zhang
}

\section{To cite this version:}

Guy Royer, H.F. Zhang. Alpha decay potential barriers and half-lives and analytical formula predictions for superheavy nuclei. Workshop on the State of the Art in Nuclear Cluster Physics (SOTANCP2008), May 2008, Strasbourg, France. pp.2270-2274, 10.1142/S021830130801146X . in2p3-00356024

\section{HAL Id: in2p3-00356024 https://hal.in2p3.fr/in2p3-00356024}

Submitted on 26 Jan 2009

HAL is a multi-disciplinary open access archive for the deposit and dissemination of scientific research documents, whether they are published or not. The documents may come from teaching and research institutions in France or abroad, or from public or private research centers.
L'archive ouverte pluridisciplinaire HAL, est destinée au dépôt et à la diffusion de documents scientifiques de niveau recherche, publiés ou non, émanant des établissements d'enseignement et de recherche français ou étrangers, des laboratoires publics ou privés. 


\title{
ALPHA DECAY POTENTIAL BARRIERS AND HALF-LIVES AND ANALYTICAL FORMULA PREDICTIONS FOR SUPERHEAVY NUCLEI
}

\author{
GUY ROYER \\ Laboratoire Subatech, UMR 6457: IN2P3/CNRS-Université-Ecole des Mines, \\ 44307 Nantes Cedex 03, France, \\ royer@subatech.in2p3.fr \\ HONGFEI ZHANG \\ School of Nuclear Science and Technology, Lanzhou University, Lanzhou 730000, \\ People's Republic of China \\ zhanghongfei@impcas.ac.cn \\ Received (received date) \\ Revised (revised date)
}

\begin{abstract}
The $\alpha$ decay potential barriers are determined in the cluster-like shape path within a generalized liquid drop model including the proximity effects between the $\alpha$ particle and the daughter nucleus and adjusted to reproduce the experimental $Q_{\alpha}$. The $\alpha$ emission half-lives are determined within the WKB penetration probability. Calculations using previously proposed formulae depending only on the mass and charge of the alpha emitter and $Q_{\alpha}$ are also compared with new experimental alpha-decay half-lives. The agreement allows to provide predictions for the $\alpha$ decay half-lives of other still unknown superheavy nuclei using the $Q_{\alpha}$ determined from the 2003 atomic mass evaluation of Audi, Wapstra and Thibault.
\end{abstract}

\section{Introduction}

The synthesis of superheavy elements has advanced strongly recently ${ }^{1}$ and their main observed decay mode is $\alpha$ emission. Predictions of $\alpha$ decay half-lives of other possible superheavy nuclei are needed. The $\alpha$ decay potential barrier is often described using a finite square well for the one-body shapes plus an hyperbola for the Coulomb repulsion between the $\alpha$ particle and its daughter. An arbitrary adjustment of the parameters allows to reproduce roughly the experimental data. Here the $\alpha$ decay potential barriers are determined in the cluster-like shape path within a generalized liquid drop model ${ }^{2}$ including the proximity effects between the $\alpha$ particle and the daughter nucleus and adjusted to reproduce the experimental $Q_{\alpha}$. The $\alpha$ emission half-lives are deduced within the WKB penetration probability through these barriers. 


\section{Alpha decay potential barriers}

The potential barrier governing the $\alpha$ decay of the ${ }^{108}$ Te nucleus is displayed in Fig. 1. The introduction of a proximity energy term lowers the barrier of $5.7 \mathrm{MeV}$ and moves the top by $2.4 \mathrm{fm}$ to a more external position. The part of the barrier corresponding to one-body shapes plays a minor role ${ }^{2}$.

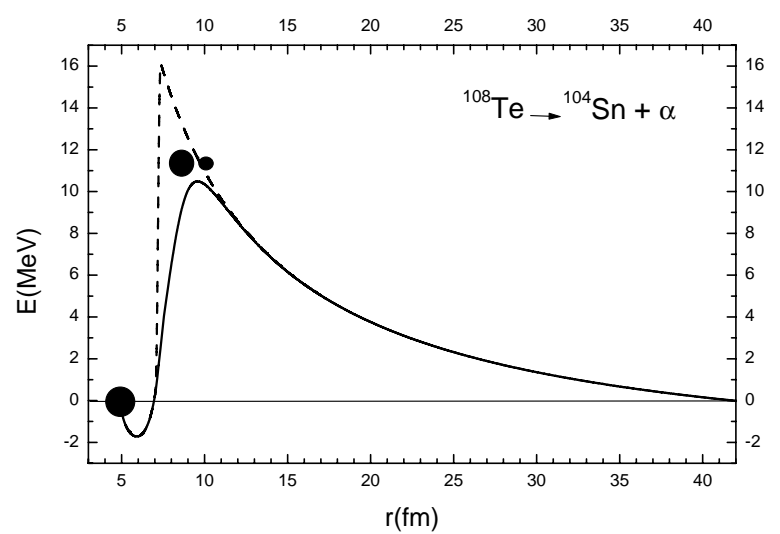

Fig. 1. Barrier against $\alpha$ decay of the ${ }^{108}$ Te mother nucleus. The broken and full curves correspond respectively to the deformation energy without and with a nuclear proximity energy term. $r$ is the centre-of-mass distance.

\section{Alpha decay half-lives}

The $\alpha$ decay half-lives have been determined assuming that the incoming point is the contact point and that the outgoing point corresponds to the equality of the Coulomb energy with the experimental $Q_{\alpha}$. The inertia parameter is simply the reduced mass. Within this unified fission model the decay constant is simply the product of the assault frequency and the penetrability. There is no preformation factor. The Fig. 2 shows that the $\alpha$ decay half-lives of the actinides and heaviest elements and their isotope dependence are correctly reproduced. For a whole set of 373 emitters the RMS deviation of $\log _{10}\left[T_{1 / 2}(s)\right]$ is only $0.63^{2,3}$.

Analytical formulae for the $\alpha$ decay half-lives have been proposed ${ }^{2}$. They lead to rms deviations of respectively $0.285,0.39,0.36$ and 0.35 for the even $(\mathrm{Z})$-even(N), even-odd, odd-even and odd-odd nuclei ${ }^{2}$.

$$
\begin{aligned}
& \log _{10}\left[T_{1 / 2}(s)\right]=-25.31-1.1629 A^{1 / 6} \sqrt{Z}+\frac{1.5864 Z}{\sqrt{Q_{\alpha}}}, \\
& \log _{10}\left[T_{1 / 2}(s)\right]=-26.65-1.0859 A^{1 / 6} \sqrt{Z}+\frac{1.5848 Z}{\sqrt{Q_{\alpha}}},
\end{aligned}
$$




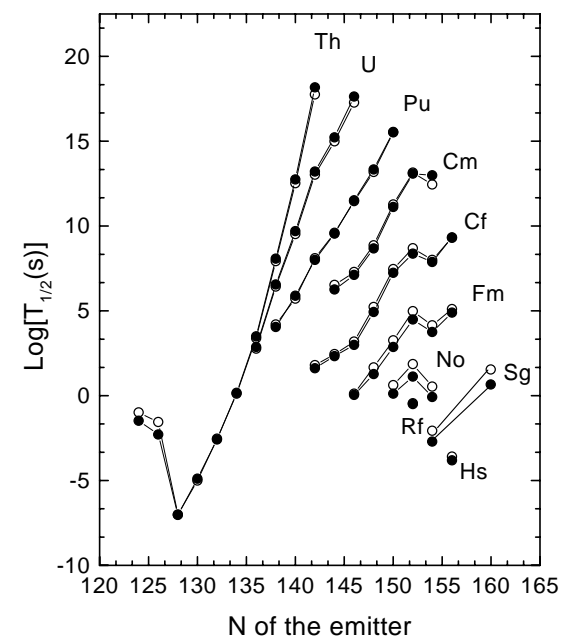

Fig. 2. Comparison between the theoretical and experimental half-lives of the heavy elements.

$$
\begin{aligned}
& \log _{10}\left[T_{1 / 2}(s)\right]=-25.68-1.1423 A^{1 / 6} \sqrt{Z}+\frac{1.592 Z}{\sqrt{Q_{\alpha}}} \\
& \log _{10}\left[T_{1 / 2}(s)\right]=-29.48-1.113 A^{1 / 6} \sqrt{Z}+\frac{1.6971 Z}{\sqrt{Q_{\alpha}}} .
\end{aligned}
$$

Later on new $\alpha$ decay half-lives have been measured and they are compared with the predictions of these formulae in the table 1 where $\mathrm{Q}$ and $\mathrm{T}$ are respectively in $\mathrm{MeV}$ and s. The good agreement allows to provide in table 2 predictions of the $\alpha$ decay half-lives of other possible superheavy elements ${ }^{4}$ with the help of the $Q_{\alpha}$ calculated from the 2003 atomic mass evaluation ${ }^{5}$. The half-live can perhaps reach one hour for the ${ }^{277} 108$ nucleus.

\section{Conclusion}

The $\alpha$ decay half-lives can be reproduced within an asymmetric fission picture and a generalized liquid drop model taking into account the proximity effects between the $\alpha$ particle and its daughter nucleus. Analytical formulae previously proposed allow to reproduce new recent data and allow to give predictions for the $\alpha$ decay half-lives of other possible superheavy elements based on the $Q_{\alpha}$ calculated from the 2003 atomic mass evaluation. 
Table 1. Comparison between recently known experimental alpha decay half-lives and results obtained with previously proposed formulae.

\begin{tabular}{|c|c|c|c|c|c|c|c|}
\hline Nucl. & $\mathrm{Q}_{\alpha}^{\exp }$ & $\mathrm{T}_{\alpha}^{\exp }$ & $\mathrm{T}_{\alpha}^{\text {Form. }}$ & Nucl. & $\mathrm{Q}_{\alpha}^{\exp }$ & $\mathrm{T}_{\alpha}^{\exp }$ & $\mathrm{T} \underset{\alpha}{F o r m .}$ \\
\hline${ }^{105} \mathrm{Te}$ & 4.900 & $0.70 \times 10^{-6}$ & $0.4 \times 10^{-6}$ & ${ }^{156} \mathrm{Er}$ & 3.486 & $2.3 \times 10^{10}$ & $1.6 \times 10^{10}$ \\
\hline${ }^{158} \mathrm{Yb}$ & 4.172 & $4.3 \times 10^{6}$ & $2.7 \times 10^{6}$ & ${ }^{160} \mathrm{Hf}$ & 4.902 & $1.9 \times 10^{3}$ & $1.9 \times 10^{3}$ \\
\hline${ }^{174} \mathrm{Hf}$ & 2.497 & $6.3 \times 10^{22}$ & $4.5 \times 10^{23}$ & ${ }^{158} \mathrm{~W}$ & 6.612 & $1.5 \times 10^{-3}$ & $1.2 \times 10^{-3}$ \\
\hline${ }^{168} \mathrm{~W}$ & 4.507 & $1.6 \times 10^{6}$ & $3.1 \times 10^{6}$ & ${ }^{162} \mathrm{Os}$ & 6.767 & $1.9 \times 10^{-3}$ & $2.3 \times 10^{-3}$ \\
\hline${ }^{164} \mathrm{Os}$ & 6.475 & $4.2 \times 10^{-2}$ & $2.2 \times 10^{-2}$ & ${ }^{166} \mathrm{Pt}$ & 7.286 & $3.0 \times 10^{-4}$ & $2.8 \times 10^{-4}$ \\
\hline${ }^{168} \mathrm{Pt}$ & 6.997 & $2.0 \times 10^{-3}$ & $2.2 \times 10^{-3}$ & ${ }^{170} \mathrm{Pt}$ & 6.708 & $1.4 \times 10^{-2}$ & $2.0 \times 10^{-2}$ \\
\hline${ }^{172} \mathrm{Hg}$ & 7.525 & $4.2 \times 10^{-4}$ & $2.7 \times 10^{-4}$ & ${ }^{174} \mathrm{Hg}$ & 7.233 & $2.1 \times 10^{-3}$ & $2.0 \times 10^{-3}$ \\
\hline${ }^{188} \mathrm{Hg}$ & 4.705 & $5.3 \times 10^{8}$ & $2.0 \times 10^{8}$ & ${ }^{178} \mathrm{~Pb}$ & 7.790 & $2.3 \times 10^{-4}$ & $2.1 \times 10^{-4}$ \\
\hline${ }^{180} \mathrm{~Pb}$ & 7.415 & $5.0 \times 10^{-3}$ & $2.7 \times 10^{-3}$ & ${ }^{184} \mathrm{~Pb}$ & 6.774 & $6.1 \times 10^{-1}$ & $3.6 \times 10^{-1}$ \\
\hline${ }^{186} \mathrm{~Pb}$ & 6.470 & $1.2 \times 10^{1}$ & $4.7 \times 10^{0}$ & ${ }^{188} \mathrm{~Pb}$ & 6.109 & $2.7 \times 10^{2}$ & $1.3 \times 10^{2}$ \\
\hline${ }^{190} \mathrm{~Pb}$ & 5.697 & $1.8 \times 10^{4}$ & $8.7 \times 10^{3}$ & ${ }^{192} \mathrm{~Pb}$ & 5.221 & $3.6 \times 10^{6}$ & $2.1 \times 10^{6}$ \\
\hline${ }^{194} \mathrm{~Pb}$ & 4.738 & $9.8 \times 10^{9}$ & $1.3 \times 10^{9}$ & ${ }^{188} \mathrm{Po}$ & 8.087 & $4.0^{\times} 10^{-4}$ & $1.1 \times 10^{-4}$ \\
\hline${ }^{189} \mathrm{Po}$ & 7.703 & $5.0 \times 10^{-3}$ & $3.0 \times 10^{-3}$ & ${ }^{190} \mathrm{Po}$ & 7.693 & $2.5 \times 10^{-3}$ & $1.5 \times 10^{-3}$ \\
\hline${ }^{192} \mathrm{Po}$ & 7.319 & $2.9 \times 10^{-2}$ & $2.2 \times 10^{-2}$ & ${ }^{210} \mathrm{Po}$ & 07 & $1.2 \times 10^{7}$ & $1.0 \times 10^{6}$ \\
\hline${ }^{196} \mathrm{Rn}$ & 7.616 & $4.4 \times 10^{-3}$ & $1.4 \times 10^{-2}$ & ${ }^{198} \mathrm{Rn}$ & 7.349 & $6.5 \times 10^{-2}$ & $9.6 \times 10^{-2}$ \\
\hline${ }^{202} \mathrm{Ra}$ & 8.020 & $2.6 \times 10^{-3}$ & $3.6 \times 10^{-3}$ & ${ }^{204} \mathrm{Ra}$ & 7.636 & $5.9 \times 10^{-2}$ & $5.5 \times 10^{-2}$ \\
\hline${ }^{210} \mathrm{Th}$ & 8.053 & $1.7 \times 10^{-2}$ & $1.3 \times 10^{-2}$ & ${ }^{212} \mathrm{Th}$ & 7.952 & $3.6 \times 10^{-2}$ & $2.4 \times 10^{-2}$ \\
\hline${ }^{218} \mathrm{U}$ & 8.773 & $5.1 \times 10^{-4}$ & $4.0 \times 10^{-4}$ & ${ }^{220} \mathrm{U}$ & 10.30 & $6.0 \times 10^{-8}$ & $5.8 \times 10^{-8}$ \\
\hline${ }^{224} \mathrm{U}$ & 8.620 & $7.0 \times 10^{-4}$ & $8.2 \times 10^{-4}$ & ${ }^{226} \mathrm{U}$ & 7.701 & $5.0 \times 10^{-1}$ & $5.7 \times 10^{-1}$ \\
\hline${ }^{228} \mathrm{Pu}$ & 7.950 & $2.0 \times 10^{-1}$ & $5.1 \times 10^{-1}$ & ${ }^{230} \mathrm{Pu}$ & 7.180 & $1.0 \times 10^{2}$ & $2.7 \times 10^{2}$ \\
\hline${ }^{238} \mathrm{Cm}$ & 6.62 & $2.3 \times 10^{5}$ & $3.3 \times 10^{5}$ & ${ }^{258} \mathrm{No}$ & 8.151 & $1.2 \times 10^{2}$ & $5.4 \times 10^{1}$ \\
\hline${ }^{258} \mathrm{Rf}$ & 9.25 & $9.2 \times 10^{-2}$ & $1.0 \times 10^{-1}$ & ${ }^{260} \mathrm{Rf}$ & 8.901 & $1.0 \times 10^{0}$ & $1.0 \times 10^{0}$ \\
\hline${ }^{266} \mathrm{Hs}$ & 10.34 & $2.3 \times 10^{-3}$ & $2.1 \times 10^{-3}$ & ${ }^{270} \mathrm{Hs}$ & 9.02 & $2.2 \times 10^{1}$ & $1.0 \times 10^{1}$ \\
\hline${ }^{270} \mathrm{Ds}$ & 11.2 & $1.0 \times 10^{-4}$ & $6.7 \times 10^{-4}$ & ${ }^{271} \mathrm{Sg}$ & 8.65 & $1.4 \times 10^{2}$ & $1.1 \times 10^{2}$ \\
\hline${ }^{272} \mathrm{Bh}$ & 9.15 & $9.8 \times 10^{0}$ & $1.8 \times 10^{1}$ & ${ }^{275} \mathrm{Hs}$ & 9.44 & $1.5 \times 10^{-1}$ & $1.9 \times 10^{0}$ \\
\hline${ }^{275} \mathrm{Mt}$ & 10.48 & $9.7 \times 10^{-3}$ & $3.2 \times 10^{-3}$ & ${ }^{276} \mathrm{Mt}$ & 9.85 & $7.2 \times 10^{-1}$ & $6.5 \times 10^{-1}$ \\
\hline${ }^{279} \mathrm{Ds}$ & 9.84 & $1.8 \times 10^{-1}$ & $6.5 \times 10^{-1}$ & ${ }^{279} \mathrm{Rg}$ & 10.52 & $1.7 \times 10^{-1}$ & $1.1 \times 10^{-2}$ \\
\hline${ }^{280} \mathrm{Rg}$ & 9.87 & $3.6 \times 10^{0}$ & $3.1 \times 10^{0}$ & 282113 & 10.63 & $7.3 \times 10^{-2}$ & $4.2 \times 10^{-2}$ \\
\hline${ }^{283} 112$ & 9.67 & $4.0 \times 10^{0}$ & $9.6 \times 10^{0}$ & ${ }^{285} 112$ & 9.29 & $3.4 \times 10^{1}$ & $1.3 \times 10^{2}$ \\
\hline${ }^{283} 113$ & 10.26 & $1.0 \times 10^{-1}$ & $2.3 \times 10^{-1}$ & ${ }^{284} 113$ & 10.15 & $4.8 \times 10^{-1}$ & $2.4 \times 10^{0}$ \\
\hline${ }^{286} 114$ & 10.35 & $1.6 \times 10^{-1}$ & $1.1 \times 10^{-1}$ & ${ }^{287} 114$ & 10.16 & $5.1 \times 10^{-1}$ & $1.8 \times 10^{0}$ \\
\hline${ }^{288} 114$ & 10.09 & $8.0 \times 10^{-1}$ & $5.2 \times 10^{-1}$ & ${ }^{289} 114$ & 9.96 & $2.7 \times 10^{0}$ & $6.1 \times 10^{0}$ \\
\hline${ }^{287} 115$ & 10.74 & $3.2 \times 10^{-2}$ & $5.3 \times 10^{-2}$ & ${ }^{288} 115$ & 10.61 & $8.7 \times 10^{-2}$ & $5.8 \times 10^{-1}$ \\
\hline${ }^{290} 116$ & 11.00 & $1.5 \times 10^{-2}$ & $8.9 \times 10^{-3}$ & ${ }^{291} 116$ & 10.89 & $6.3 \times 10^{-3}$ & $8.9 \times 10^{-2}$ \\
\hline${ }^{292} 116$ & 10.80 & $1.8 \times 10^{-2}$ & $2.7 \times 10^{-2}$ & ${ }^{293} 116$ & 10.67 & $5.3 \times 10^{-2}$ & $3.1 \times 10^{-1}$ \\
\hline${ }^{294} 118$ & 11.81 & $1.8^{\times} 10^{-3}$ & $3.9^{\times} 10^{-4}$ & & & & \\
\hline
\end{tabular}

\section{References}

1. Yu. Ts. Oganessian et al., Phys. Rev. C76 (2007) 011601(R).

2. G. Royer, J. Phys. G: Nucl. Part. Phys. 26 (2000) 1149.

3. R. Moustabchir and G. Royer, Nucl. Phys. A683 (2001) 266.

4. G. Royer and H. F. Zhang, Phys. Rev. C77 (2008) 037602.

5. G. Audi, A. H. Wapstra, anc C. Thibault, Nucl. Phys. A729 (2003) 337. 
Table 2. Predicted $\alpha$ decay half-lives using the formulae (1-4).

\begin{tabular}{|c|c|c|c|c|c|c|c|c|}
\hline${ }_{Z}^{A}$ & Q & $T_{1 / 2}^{f o r m}$ & $\stackrel{A}{Z}$ & Q & $T_{1 / 2}^{\text {form }}$ & $\stackrel{A}{Z}$ & Q & $T_{1 / 2}^{\text {form }}$ \\
\hline 118 & 12.30 & $187 \mu \mathrm{s}$ & $\begin{array}{l}292 \\
117\end{array}$ & 11.60 & $6.47 \mathrm{~ms}$ & $\begin{array}{l}291 \\
117\end{array}$ & 11.90 & $0.32 \mathrm{~ms}$ \\
\hline 1180 & 10.00 & $4.8 \mathrm{~s}$ & $\begin{array}{l}217 \\
290 \\
115\end{array}$ & 10.30 & $4.2 \mathrm{~s}$ & $\begin{array}{l}289 \\
116\end{array}$ & 11.70 & $1.05 \mathrm{~ms}$ \\
\hline & 10.60 & $113 \mathrm{~ms}$ & $\begin{array}{l}219 \\
113\end{array}$ & 9.34 & $99.4 \mathrm{~s}$ & $\begin{array}{l}210 \\
113\end{array}$ & 9.68 & $61.5 \mathrm{~s}$ \\
\hline & 11.00 & $12 \mathrm{~ms}$ & $\begin{array}{l}213 \\
285 \\
113\end{array}$ & 10.02 & $1.0 \mathrm{~s}$ & $\begin{array}{l}213 \\
284 \\
112\end{array}$ & 9.30 & $25.1 \mathrm{~s}$ \\
\hline & 8.96 & $5.5 \mathrm{~min}$ & $\begin{array}{l}282 \\
112\end{array}$ & 9.96 & $0.297 \mathrm{~s}$ & & 9.38 & $99.8 \mathrm{~s}$ \\
\hline & 10.28 & $0.2 \mathrm{~s}$ & $\begin{array}{l}281 \\
111\end{array}$ & 9.64 & $2.72 \mathrm{~s}$ & $\begin{array}{l}281 \\
110\end{array}$ & 8.96 & $4.6 \mathrm{~min}$ \\
\hline & 10.62 & $25.4 \mathrm{~ms}$ & $\begin{array}{l}280 \\
111\end{array}$ & 9.98 & $1.43 \mathrm{~s}$ & $\begin{array}{l}2799 \\
112\end{array}$ & 10.96 & $3.88 \mathrm{~ms}$ \\
\hline lo & 8.70 & $7.72 \mathrm{~min}$ & $\begin{array}{l}278 \\
112\end{array}$ & 11.38 & $0.083 \mathrm{~ms}$ & $\begin{array}{l}278 \\
111\end{array}$ & 10.72 & $12.5 \mathrm{~ms}$ \\
\hline 1 & 10.00 & $51.8 \mathrm{~ms}$ & $\begin{array}{l}278 \\
109\end{array}$ & 9.10 & $143 \mathrm{~s}$ & $\begin{array}{l}277 \\
112 \\
112 \\
\end{array}$ & 11.62 & $0.12 \mathrm{~ms}$ \\
\hline & 11.18 & $0.28 \mathrm{~ms}$ & $\begin{array}{l}277 \\
110\end{array}$ & 10.30 & $39 \mathrm{~ms}$ & $\begin{array}{l}277 \\
277 \\
109\end{array}$ & 9.50 & $1.48 \mathrm{~s}$ \\
\hline & 8.40 & $65.25 \mathrm{~min}$ & $\begin{array}{l}276 \\
111\end{array}$ & 11.32 & $0.39 \mathrm{~ms}$ & $\begin{array}{l}276 \\
110\end{array}$ & 10.60 & $1.47 \mathrm{~ms}$ \\
\hline & 8.80 & $40.6 \mathrm{~s}$ & & 11.55 & $42.3 \mu \mathrm{s}$ & $\begin{array}{l}275 \\
110\end{array}$ & 11.10 & $0.43 \mathrm{~ms}$ \\
\hline $\begin{array}{l}274 \\
111\end{array}$ & 11.60 & $88.1 \mu \mathrm{s}$ & $\begin{array}{l}274 \\
110\end{array}$ & 11.40 & $19.5 \mu \mathrm{s}$ & $\begin{array}{l}2774 \\
109\end{array}$ & 10.50 & $9.84 \mathrm{~ms}$ \\
\hline $\begin{array}{l}274 \\
108\end{array}$ & 9.50 & $0.3 \mathrm{~s}$ & $\begin{array}{l}274 \\
107\end{array}$ & 8.50 & $48.45 \min$ & $\begin{array}{l}273 \\
111\end{array}$ & 11.20 & $0.29 \mathrm{~ms}$ \\
\hline $\begin{array}{l}273 \\
\end{array}$ & 11.37 & $0.11 \mathrm{~ms}$ & $\begin{array}{l}173 \\
273 \\
109\end{array}$ & 10.82 & $0.5 \mathrm{~ms}$ & $\begin{array}{l}1713 \\
2708 \\
108\end{array}$ & 9.90 & $101 \mathrm{~ms}$ \\
\hline & 8.90 & $21.1 \mathrm{~s}$ & $\begin{array}{l}272 \\
110\end{array}$ & 10.76 & $0.697 \mathrm{~ms}$ & $\begin{array}{l}272 \\
109 \\
109\end{array}$ & 10.60 & $5.74 \mathrm{~ms}$ \\
\hline 27 & 10.10 & $6.9 \mathrm{~ms}$ & $\begin{array}{l}272 \\
106\end{array}$ & 8.30 & $6.38 \mathrm{~min}$ & $\begin{array}{l}271 \\
110\end{array}$ & 10.87 & $1.79 \mathrm{~ms}$ \\
\hline & 10.14 & $29.9 \mathrm{~ms}$ & $\begin{array}{l}271 \\
108\end{array}$ & 9.90 & $109.7 \mathrm{~ms}$ & $\begin{array}{l}271 \\
107\end{array}$ & 9.50 & $0.338 \mathrm{~s}$ \\
\hline & 11.20 & $0.067 \mathrm{~ms}$ & $\begin{array}{l}270 \\
109\end{array}$ & 10.35 & $30 \mathrm{~ms}$ & $\begin{array}{l}270 \\
108\end{array}$ & 9.30 & $1.4 \mathrm{~s}$ \\
\hline & 9.30 & $6.25 \mathrm{~s}$ & $\begin{array}{l}270 \\
106\end{array}$ & 9.10 & $0.99 \mathrm{~s}$ & $\begin{array}{l}270 \\
105\end{array}$ & 8.20 & $94.58 \mathrm{~min}$ \\
\hline & 10.53 & $3.12 \mathrm{~ms}$ & $\begin{array}{l}269 \\
108\end{array}$ & 9.63 & $0.68 \mathrm{~s}$ & $\begin{array}{l}269 \\
107\end{array}$ & 8.84 & $39 \mathrm{~s}$ \\
\hline & 8.80 & $37.5 \mathrm{~s}$ & $\begin{array}{l}269 \\
105\end{array}$ & 8.40 & $3.01 \mathrm{~min}$ & & 11.92 & $1.84 \mu \mathrm{s}$ \\
\hline & 10.73 & $3.07 \mathrm{~ms}$ & $\begin{array}{l}268 \\
108\end{array}$ & 9.90 & $28.6 \mathrm{~ms}$ & $\begin{array}{l}268 \\
107\end{array}$ & 9.08 & $35.4 \mathrm{~s}$ \\
\hline 26 & 8.40 & $3.4 \mathrm{~min}$ & $\begin{array}{l}268 \\
105\end{array}$ & 8.20 & $102.7 \mathrm{~min}$ & $\begin{array}{l}268 \\
104\end{array}$ & 8.10 & $5.88 \mathrm{~min}$ \\
\hline 26 & 12.28 & $1.57 \mu \mathrm{s}$ & $\begin{array}{l}267 \\
109\end{array}$ & 10.87 & $0.49 \mathrm{~ms}$ & $\begin{array}{l}267 \\
108\end{array}$ & 10.12 & $32.9 \mathrm{~ms}$ \\
\hline $\begin{array}{l}26 \\
10\end{array}$ & 9.37 & $0.97 \mathrm{~s}$ & $\begin{array}{l}267 \\
106\end{array}$ & 8.64 & $2.25 \mathrm{~min}$ & $\begin{array}{l}267 \\
105\end{array}$ & 7.90 & $205 \mathrm{~min}$ \\
\hline 26 & 7.80 & $306 \min$ & $\begin{array}{l}266 \\
109\end{array}$ & 10.996 & $0.69 \mathrm{~ms}$ & $\begin{array}{l}266 \\
108\end{array}$ & 10.336 & $2.16 \mathrm{~ms}$ \\
\hline $\begin{array}{l}266 \\
107\end{array}$ & 9.55 & $1.21 \mathrm{~s}$ & $\begin{array}{l}266 \\
105\end{array}$ & 8.19 & $121.8 \mathrm{~min}$ & $\begin{array}{l}266 \\
104\end{array}$ & 7.50 & $20.09 \mathrm{~h}$ \\
\hline $\begin{array}{l}265 \\
109\end{array}$ & 11.07 & $0.178 \mathrm{~ms}$ & $\begin{array}{l}265 \\
107\end{array}$ & 9.77 & $74.4 \mathrm{~ms}$ & $\begin{array}{l}265 \\
105\end{array}$ & 8.49 & $1.76 \mathrm{~min}$ \\
\hline $\begin{array}{l}265 \\
104\end{array}$ & 7.78 & $6.58 \mathrm{~h}$ & $\begin{array}{l}264 \\
107\end{array}$ & 9.97 & $74.1 \mathrm{~ms}$ & $\begin{array}{l}264 \\
106\end{array}$ & 9.21 & $0.60 \mathrm{~s}$ \\
\hline $\begin{array}{l}264 \\
105\end{array}$ & 8.66 & $154 \mathrm{~s}$ & $\begin{array}{l}264 \\
104\end{array}$ & 8.14 & $5.03 \mathrm{~min}$ & $\begin{array}{l}263 \\
108\end{array}$ & 10.67 & $1.52 \mathrm{~ms}$ \\
\hline 263 & 10.08 & $11.6 \mathrm{~ms}$ & $\begin{array}{l}263 \\
105\end{array}$ & 9.01 & $2.4 \mathrm{~s}$ & $\begin{array}{l}263 \\
104\end{array}$ & 8.49 & $76.8 \mathrm{~s}$ \\
\hline 107 & 10.30 & $9.51 \mathrm{~ms}$ & $\begin{array}{l}262 \\
106\end{array}$ & 9.60 & $47.5 \mathrm{~ms}$ & $\begin{array}{l}262 \\
105\end{array}$ & 9.01 & $10.9 \mathrm{~s}$ \\
\hline $\begin{array}{l}262 \\
104\end{array}$ & 8.49 & $20.6 \mathrm{~s}$ & $\begin{array}{l}261 \\
107\end{array}$ & 10.56 & $0.74 \mathrm{~ms}$ & $\begin{array}{l}261 \\
106\end{array}$ & 9.80 & $56.1 \mathrm{~ms}$ \\
\hline $\begin{array}{l}261 \\
105 \\
105\end{array}$ & 9.22 & $0.60 \mathrm{~s}$ & $\begin{array}{l}260 \\
107\end{array}$ & 10.47 & $3.58 \mathrm{~ms}$ & 104 & 8.90 & $1.08 \mathrm{~s}$ \\
\hline 106 & 9.83 & $50.5 \mathrm{~ms}$ & $\begin{array}{l}259 \\
105\end{array}$ & 9.62 & $45.9 \mathrm{~ms}$ & $\begin{array}{l}259 \\
104\end{array}$ & 9.12 & $0.93 \mathrm{~s}$ \\
\hline 106 & 9.67 & $36.1 \mathrm{~ms}$ & $\begin{array}{l}258 \\
105\end{array}$ & 9.48 & $0.42 \mathrm{~s}$ & $\begin{array}{l}258 \\
104\end{array}$ & 9.25 & $103 \mathrm{~ms}$ \\
\hline & 9.23 & $0.67 \mathrm{~s}$ & $\begin{array}{l}257 \\
104\end{array}$ & 9.04 & $1.76 \mathrm{~s}$ & $\begin{array}{l}256 \\
105\end{array}$ & 9.46 & $522 \mathrm{~ms}$ \\
\hline 104 & 8.93 & $1.04 \mathrm{~s}$ & $\begin{array}{l}255 \\
105\end{array}$ & 9.72 & $28.9 \mathrm{~ms}$ & $\begin{array}{l}255 \\
104\end{array}$ & 9.058 & $1.69 \mathrm{~s}$ \\
\hline
\end{tabular}

